\title{
Primary Hepatic Synovial Sarcoma with \\ Synchronous Colorectal Adenocarcinoma: A Case Report and Review of Literature
}

\author{
Xuan Vinh Vu \\ Bach Mai Hospital \\ Thanh Khiem Nguyen \\ Bach Mai Hospital \\ Ham Hoi Nguyen \\ Bach Mai Hospital \\ Ngoc Hung Nguyen \\ Bach Mai Hospital \\ Tuan Hiep Luong ( $\square$ hiep1995hsgs@gmail.com ) \\ Hanoi Medical University \\ Cong Nguyen Nguyen \\ Bach Mai Hospital \\ Van Chuong Tran \\ Bach Mai Hospital
}

Case Report

Keywords: Synovial sarcoma, Primary hepatic, Case report

Posted Date: February 24th, 2022

DOI: https://doi.org/10.21203/rs.3.rs-1374869/v1

License: (c) (i) This work is licensed under a Creative Commons Attribution 4.0 International License.

Read Full License 


\section{Abstract}

Background: Primary hepatic synovial sarcoma is an extremely rare disease with a poor prognosis. The symptoms are nonspecific, and it is usually diagnosed at an advanced stage.

Case presentation: A 63-year-old male patient was admitted with asymptomatic but elevated Alpha Fetoprotein (AFP) serum level in routine medical examination with a medical history of Hepatitis B with Tenofovir $300 \mathrm{mg}$ per day. No sign of jaundice was observed. Her abdomen was flat and soft without tenderness or palpable mass. Dynamic abdominal computed tomography (CT) scan and magnetic resonance imaging (MRI) showed a hypervascular $15 \times 26 \mathrm{~mm}$ mass within hepatic subsegment $\mathrm{V}$. There was no distant spread location. Colonoscopy revealed 3-cm sessile polyp in Sigmoid colon with pathological result was adenocarcinoma. Immunohistochemistry was performed to accurate diagnosis. The tumor cells were positive for CD99, EMA and a Ki67 value of $40 \%$ and were negative for Hepar-1, CD34, CD117, CK7, STAT6, DOG1, SMA and S100. The final diagnosis for this patient was a Malignant Sigmoid-colon polyp with a Primary hepatic synovial sarcoma. Laparoscopic Sigmoid-colon and rectal resection with injecting autologous blood to mark the tumor before surgery was performed for malignant polypectomy lesion and Hepatic Radiofrequency Ablation (RFA) was performed four days before surgery. With no postoperative complications as well as acute hepatic failure, the patient was discharged on the POD8 uneventfully.

Conclusion: A primary hepatic synovial sarcoma is especially rare, with the present case being the fifth accounted in the English literature. Synovial sarcoma (SS) is a genetically soft tissue sarcoma with uncertain differentiation and originated from primitive mesenchymal cells. We here present a report of this case with had primary hepatic synovial sarcoma synchronously with a colorectal adenocarcinoma.

\section{Background}

Synovial sarcomas (SS) are rare, aggressive sarcomas of primitive mesenchymal cells, set in a hemangiopericytoma (HPC)-like vascular pattern, which account for $5 \%-10 \%$ of all soft tissue sarcoma [1, 2]. Synovial sarcomas can be found in any part of the body, primarily arising in the extremities, especially in the anatomic structures adjacent to the knee joint, but extremely rare in the abdominal viscera, especially the liver, with only a few cases reported in the literature [3-7]. The specific etiology of synovial sarcoma remains unknown. Several risk factors have been associated with poor prognosis, including tumor size, anatomic visceral sites as well as low-graded [8-10]. The clinical signs and symptoms of hepatic synovial sarcoma are nonspecific. Survival outcome was occasionally poor, with the five-year survival rate is about $50-60 \%[11-13]$. We herein present a report of a primary hepatic synovial sarcoma with a Malignant Sigmoid colon polyp. This report can be useful for both education and clinical practice purposes. All our work has been reported in line with the CARE criteria and guidelines [14].

\section{Case Presentation}


A 63-year-old male patient was admitted with asymptomatic but elevated Alpha Fetoprotein (AFP) serum level in routine medical examination. He had a medical history of Hepatitis B with Tenofovir $300 \mathrm{mg}$ per day. He denied current or prior alcohol, smoking or with no known exposure to carcinogens and drugs. Physical examination showed clinical signs within normal limits. No sign of jaundice was observed. Her abdomen was flat and soft without tenderness or palpable mass. The AFP serum level was $43.5 \mathrm{ng} / \mathrm{ml}$. Dynamic abdominal computed tomography (CT) scan showed a heterogenous $15 \times 26 \mathrm{~mm}$ mass within hepatic subsegments IV-V. Similarly, Magnetic resonance imaging (MRI) showed a heterogenous mass with low and high intensity on T1 and T2-weighted images with post-gadolinium enhancement, respectively. There was no distant spread location (Fig. 1). Colonoscopy revealed 3-cm sessile polyp in Sigmoid colon with pathological result was adenocarcinoma. A percutaneous liver biopsy fine needle aspirations (FNA) was proceeded, and the result was Spindle cell tumor. Immunohistochemistry was performed to accurate diagnosis. The tumor cells were positive for CD99, EMA and a Ki67 value of 40\% and were negative for Hepar-1, CD34, CD117, CK7, STAT6, D0G1, SMA and S100 (Fig. 2). The final pathological result was a Hepatic primary synovial sarcoma. The final diagnosis for this patient was a Malignant sigmoid-colon polyp with a Primary hepatic synovial sarcoma. MDT meeting consisted of surgeons, physicians, clinical and medical oncologists, radiologists, pathologists, and clinical nurse specialists (CNSs) were organized to make clinical decisions. The informed consent was signed.

Laparoscopic Sigmoid-colon and rectal resection with injecting autologous blood to mark the tumor before surgery was performed for malignant polypectomy lesion. Four days before surgery, Hepatic Radiofrequency Ablation (RFA) was performed for Hepatic primary synovial sarcoma. With no postoperative complications as well as acute hepatic failure, the patient was discharged on the POD8 uneventfully. At the most recent 6 months follow-up after the operation, he was still alive without local recurrence and distant metastases were identified.

\section{Discussion And Conclusion}

Synovial sarcoma (SS) is a genetically soft tissue sarcoma with uncertain differentiation, with originate from primitive mesenchymal cells, not from intra-articular synovium like its misnomer $[1,13]$. Despite of being a rare malignancy sarcoma with an incidence of 800 to 1,000 cases per year in the United States, adolescents and adults younger than 30 years of age were believed the most common but favorable prognostic group of patients [15]. In location, most primary tumors were found near articular structures, especially in the lower extremities [1]. However, primary synovial sarcomas which was raised from uncommon locations such as mediastinum, retroperitoneum, and various viscera has been reported [1, 2, 13]. To our best knowledge, there were only 4 cases of primary hepatic synovial sarcoma which have been reported in the English-language literature [3-6]. The age of the 4 reported cases was 13, 18, 44, and 60 years, respectively. So that, with a case of hepatic spindle cell tumors was found, the possibility of metastases from extrahepatic sarcomas should always be excluded [3]. In our case, all imaging and physical examinations have excluded the possibility of secondary tumor.

In imaging characteristics, liked all other soft-tissue sarcomas, magnetic resonance imaging (MRI) is the gold standard for diagnostic imaging, with typically well-defined and heterogenous solid mass with low 
and high intensity on T1 and T2-weighted images with post-gadolinium enhancement, respectively [16]. Computed tomography (CT) with contrast can be applied for cases with untypical results in MRI. With cases of liver metastasis, on CT, SS had hypodense appearance comparing with muscle as well as heterogeneous enhancement with calcifications [17]. In our cases, all MRI and CT were used, and the tumor has appeared with similar appearances. Due to the patient's medical history of Hepatitis B and elevated Alpha Fetoprotein (AFP) serum level in routine medical examination, the most-likely diagnosis was Hepatocellular carcinoma (HCC), but it hadn't met the criteria of the 2018 ESMO Clinical Practice Guidelines for diagnosis in Imaging characteristics and Alpha Fetoprotein (AFP) serum level [18], so that, we have proceeded to take a percutaneous liver biopsy with fine needle aspirations (FNA) and pathologic assessment for accurate diagnosis.

Microscopically and histologically, all the 5 cases (including the current one) exhibited classic features of synovial sarcoma with spindle cells. That represents for the monomorphic appearance, and other two sub-types were biphasic (associated with variable epithelial differentiation) or poorly differentiated (with greater nuclear atypia and high mitotic activity) [1]. On immunohistochemistry, about $60 \%$ of all cases, tumoral cells were positive for CD99, as well as always negative for CD34 [19]. In our case, the tumor stain positive focally for Epithelial Membrane Antigen (EMA), represented epithelial differentiation in this case. So that, we have consumed that our tumor was biphasic sub-type. In molecular pathology, a pathognomonic translocation between chromosomes $X$ and 18 , or $t(X ; 18)(p 11.2 ; q 11.2)$, was characterized for SS, with an rate of over $95 \%$ of cases, with can be identified by exact fusion transcript (SS18-SS X1) $[13,20]$. Due to its rarity, the differential diagnosis of primary hepatic synovial sarcoma is quite broad, with other more common types of primary liver sarcomas, such as hemangioendothelioma, angiosarcoma, leiomyosarcoma, embryonal rhabdomyosarcoma, carcinosarcoma and giant cell sarcoma [21]. In our case, the tumor stain negative for Hepar-1, DOG1, CD117, STAT6, CD34, SMA and S100. So that, we can occasionally rule out the possibility of hepatocellular carcinoma, gastrointestinal stromal tumor, other types of solitary fibrous tumors [22-24].

And due to the rarity of primary liver sarcomas (PLS), especially primary hepatic SS, the optimal treatment approach is still not well-defined, which was depended on histological type and the ability to accomplish negative resection margins[21, 25]. In our case, with tumoral position deeply within hepatic subsegments IV-V and near important anatomical landmarks that are right and middle hepatic veins, as well as small size (about $2 \mathrm{~cm}$ ), we had decided to proceed Hepatic Radiofrequency Ablation (RFA), due to its utility of local tumor control with minimal morbidity in patients with small-sized hepatic tumors compared to liver resection [26]. However, with high-rated of local recurrence and distant metastasis, especially in elderly populations and high Ki-67 index, primary synovial sarcoma must be follow-up carefully for early detection and management [1].

\section{Conclusion}

To the best of our knowledge, the present patient is the fifth case report of primary hepatic synovial sarcoma. Imaging features are non-specific, with the diagnosis being only established through histology 
and immunohistochemistry analysis. Our discussion emphasizes the difficulty associated with diagnosis and management of this rare hepatic neoplasm.

\section{Abbreviations}

CD: Cluster of differentiation; CK: Cyclin-dependent kinase; CT: Computed tomography; DOG1: Discovered on GIST-1; MRI: Magnetic resonance imaging; RFA: Radiofrequency Ablation; STAT6: Signal transducer and activator of transcription 6, EMA: Epithelial membrane antigen; SMA: Smooth muscle actin.

\section{Declarations}

\section{Ethics approval and consent to participate}

Ethics approval of this study was given by the Research Ethics Committees of Bach Mai Hospital. Authors have agreed to submit it in its current form for consideration for publication in the Journal.

\section{Consent for publication}

Written informed consent for publication of their clinical details and clinical images was obtained from the patient's next of kin.

\section{Availability of data and materials}

All data generated or analyzed during this study are included in this published article.

\section{Competing interests}

The authors declare that there is no conflict of interest regarding the publication of this article.

\section{Funding}

The authors declare no funding for this study.

\section{Authors' contributions}

$\mathrm{XVV}$ and TKN contributed equally as co-first authors, the main doctors conceived the original idea and operated the patients; $\mathrm{HHN}$ and NHN edited the manuscript; THL performed the operation and wrote the manuscript; CNN performed the operation; VCT edited the manuscript. The co-first authors and the other authors discussed the results together and contributed to the final manuscript. All authors read and approved the final manuscript.

\section{Acknowledgements}

The authors wish to thank the board of the Department of Gastrointestinal and Hepato-pancreato-biliary surgery, Bach Mai Hospital, Hanoi, Vietnam for their assistance during the time in-hospital observation of 
this patient.

Author details

${ }^{1}$ Department of Gastrointestinal and Hepato-pancreato-biliary surgery, Bach Mai Hospital, Hanoi, Vietnam. ${ }^{2}$ Department of Surgery, Hanoi Medical University, Vietnam. ${ }^{3}$ Pathology Center, Bach Mai Hospital, Hanoi, Vietnam

\section{References}

1. Gazendam AM, Popovic S, Munir S, Parasu N, Wilson D, Ghert M: Synovial Sarcoma: A Clinical Review. Current oncology (Toronto, Ont) 2021, 28(3):1909-1920.

2. Romeo S, Rossi S, Acosta Marín M, Canal F, Sbaraglia M, Laurino L, Mazzoleni G, Montesco MC, Valori L, Campo Dell'Orto M et al: Primary Synovial Sarcoma (SS) of the digestive system: a molecular and clinicopathological study of fifteen cases. Clin Sarcoma Res 2015, 5:7-7.

3. Holla P, Hafez GR, Slukvin I, Kalayoglu M: Synovial sarcoma, a primary liver tumor-a case report. Pathology, research and practice 2006, 202(5):385-387.

4. Janevska V, Filipovski V, Banev S, Janevski V, Jovcevski A, Spasevska L, Rubens J, KostadinovaKunovska S, Krsteska B, Janevska C et al: Synovial Sarcoma of the Liver-A Case Report. Jun Macedonian Journal of Medical Sciences Jun Maced J Med Sci Jun 2011, 15:185-191185.

5. Srivastava A, Nielsen PG, Cin PD, Rosenberg AE: Monophasic Synovial Sarcoma of the Liver. Archives of Pathology \& Laboratory Medicine 2005, 129(8):1047-1049.

6. Xiong B, Chen M, Ye F, Zhang Z, Yin L, Huang H, Chen H, Zhang H: Primary Monophasic Synovial Sarcoma of the Liver in a 13-Year-Old Boy. Pediatric and Developmental Pathology 2013, 16(5):353-356.

7. Zimmermann A: Synovial-Like Neoplasms (Synovial Sarcoma) of the Liver. In: Tumors and Tumor-Like Lesions of the Hepatobiliary Tract. edn. Cham: Springer International Publishing; 2016: 1-9.

8. Callegaro D, Miceli R, Bonvalot S, Ferguson P, Strauss DC, Levy A, Griffin A, Hayes AJ, Stacchiotti S, Pechoux CL et al: Development and external validation of two nomograms to predict overall survival and occurrence of distant metastases in adults after surgical resection of localised soft-tissue sarcomas of the extremities: a retrospective analysis. The Lancet Oncology 2016, 17(5):671-680.

9. Ferrari A, Bisogno G, Alaggio R, Cecchetto G, Collini P, Rosolen A, Meazza C, Indolfi P, Garaventa A, De Sio L et al: Synovial sarcoma of children and adolescents: the prognostic role of axial sites. European journal of cancer (Oxford, England : 1990) 2008, 44(9):1202-1209.

10. Soft tissue and visceral sarcomas: ESMO Clinical Practice Guidelines for diagnosis, treatment and follow-up. Annals of oncology : official journal of the European Society for Medical Oncology 2014,25 
Suppl 3:iii102-112.

11. Trassard M, Le Doussal V, Hacène $K$, Terrier P, Ranchère $D$, Guillou L, Fiche $M$, Collin F, Vilain MO, Bertrand $\mathrm{G}$ et al: Prognostic factors in localized primary synovial sarcoma: a multicenter study of 128 adult patients. Journal of clinical oncology : official journal of the American Society of Clinical Oncology 2001, 19(2):525-534.

12. ten Heuvel SE, Hoekstra HJ, Bastiaannet E, Suurmeijer AJ: The classic prognostic factors tumor stage, tumor size, and tumor grade are the strongest predictors of outcome in synovial sarcoma: no role for SSX fusion type or ezrin expression. Applied immunohistochemistry \& molecular morphology : AIMM 2009, 17(3):189-195.

13. Stacchiotti S, Tine BAV: Synovial Sarcoma: Current Concepts and Future Perspectives. Journal of Clinical Oncology 2018, 36(2):180-187.

14. Gagnier JJ, Kienle G, Altman DG, Moher D, Sox H, Riley D, Group* C: The CARE Guidelines: Consensusbased Clinical Case Reporting Guideline Development. Glob Adv Health Med 2013, 2(5):38-43.

15. Ferrari A, De Salvo GL, Brennan B, van Noesel MM, De Paoli A, Casanova M, Francotte N, Kelsey A, Alaggio R, Oberlin $O$ et al: Synovial sarcoma in children and adolescents: the European Pediatric Soft Tissue Sarcoma Study Group prospective trial (EpSSG NRSTS 2005). Annals of Oncology 2015, 26(3):567-572.

16. O'SULLIVAN PJ, HARRIS AC, MUNK PL: Radiological features of synovial cell sarcoma. The British Journal of Radiology 2008, 81(964):346-356.

17. Baheti AD, Tirumani SH, Sewatkar R, Shinagare AB, Hornick JL, Ramaiya NH, Jagannathan JP: Imaging features of primary and metastatic extremity synovial sarcoma: a single institute experience of 78 patients. The British Journal of Radiology 2015, 88(1046):20140608.

18. Vogel A, Cervantes A, Chau I, Daniele B, Llovet JM, Meyer T, Nault JC, Neumann U, Ricke J, Sangro B et al: Hepatocellular carcinoma: ESMO Clinical Practice Guidelines for diagnosis, treatment and follow-up. Annals of oncology : official journal of the European Society for Medical Oncology 2018, 29(Suppl 4):iv238-iv255.

19. Moch H: Soft Tissue and Bone Tumours WHO Classification of Tumours/Volume 3. WHO Classification of Tumours 2020, 3.

20. Stegmaier S, Leuschner I, Poremba C, Ladenstein R, Kazanowska B, Ljungman G, Scheer M, Blank B, Bielack S, Klingebiel T et al: The prognostic impact of SYT-SSX fusion type and histological grade in pediatric patients with synovial sarcoma treated according to the CWS (Cooperative Weichteilsarkom Studie) trials. Pediatric blood \& cancer 2017, 64(1):89-95. 
21. Konstantinidis IT, Nota C, Jutric Z, Ituarte P, Chow W, Chu P, Singh G, Warner SG, Melstrom LG, Fong Y: Primary liver sarcomas in the modern era: Resection or transplantation? J Surg Oncol 2018, 117(5):886891.

22. Yoshida A, Tsuta K, Ohno M, Yoshida M, Narita Y, Kawai A, Asamura H, Kushima R: STAT6 immunohistochemistry is helpful in the diagnosis of solitary fibrous tumors. The American journal of surgical pathology 2014, 38(4):552-559.

23. Hanif R, Mansoor S: Hep par-1: a novel immunohistochemical marker for differentiating hepatocellular carcinoma from metastatic carcinoma. Journal of the College of Physicians and Surgeons-Pakistan : JCPSP 2014, 24(3):186-189.

24. Lopes LF, West RB, Bacchi LM, van de Rijn M, Bacchi CE: DOG1 for the diagnosis of gastrointestinal stromal tumor (GIST): Comparison between 2 different antibodies. Applied immunohistochemistry \& molecular morphology : AIMM 2010, 18(4):333-337.

25. Weitz J, Klimstra DS, Cymes K, Jarnagin WR, D'Angelica M, La Quaglia MP, Fong Y, Brennan MF, Blumgart LH, DeMatteo RP: Management of primary liver sarcomas. Cancer 2007, 109(7):1391-1396.

26. McDermott S, Gervais DA: Radiofrequency ablation of liver tumors. Seminars in interventional radiology $2013,30(1): 49-55$.

\section{Figures}



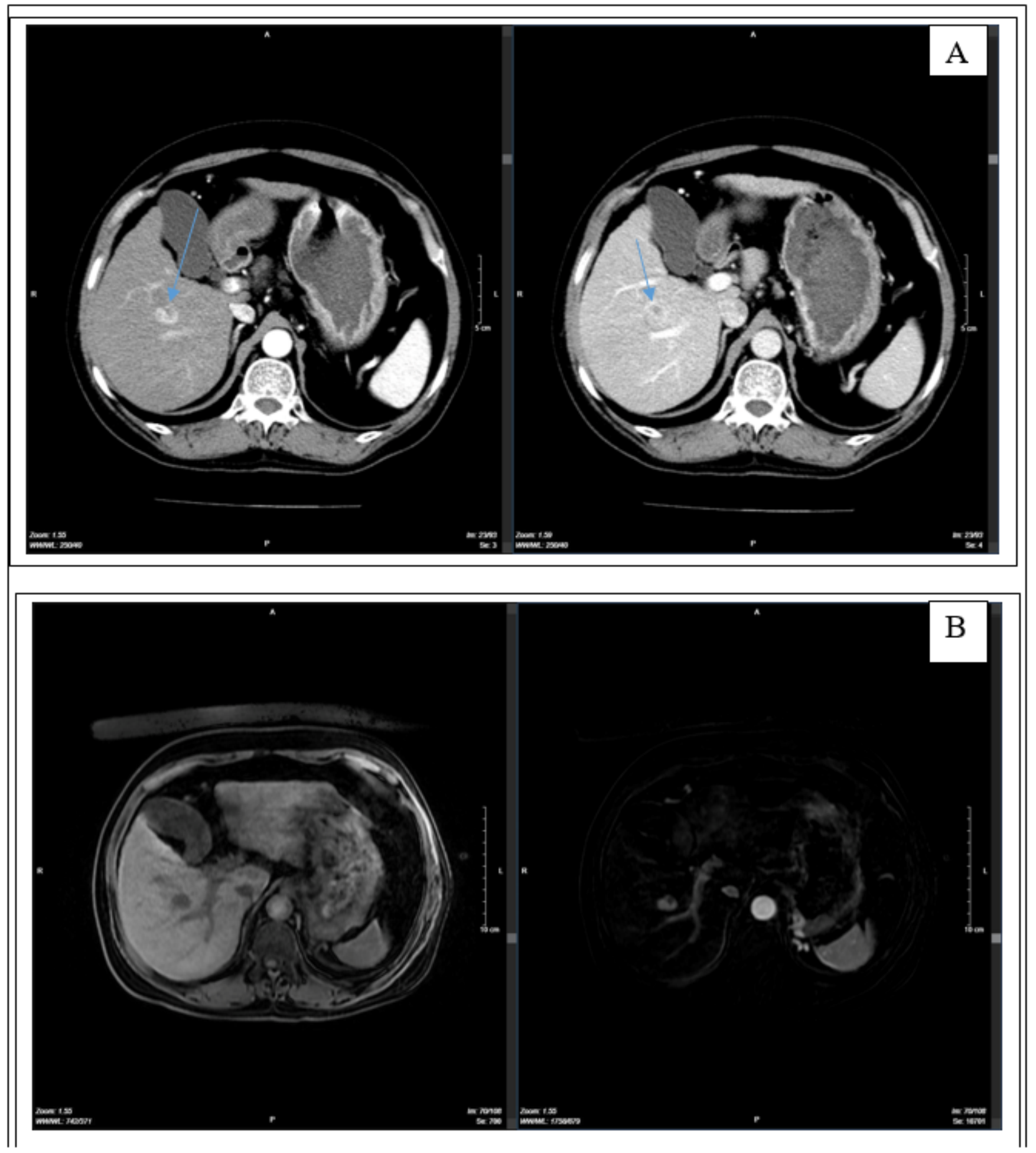

Figure 1

(A) Dynamic abdominal computed tomography (CT) scan showed a heterogenous $15 \times 26 \mathrm{~mm}$ mass within hepatic subsegment IV-V.

(B) Magnetic resonance imaging (MRI) showed a heterogenous mass with low intensity on T1 and high intensity on T2-weighted images with post-gadolinium enhancement. 


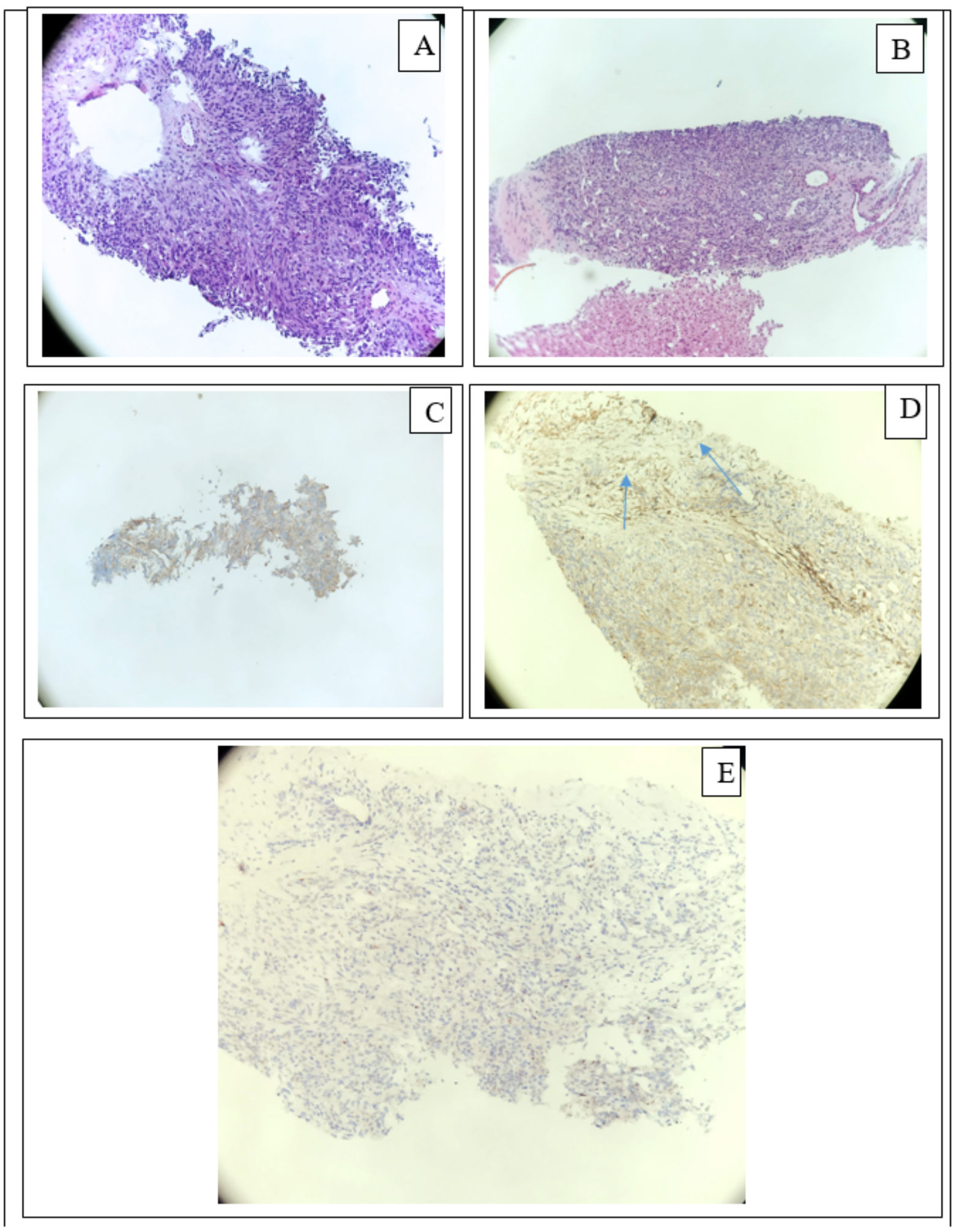

Figure 2

(A) (B) The result of hematoxylin and eosin (H\&E) and Periodic acid Schiff (PAS) staining was Spindle cell tumor. 
(C) (D) (E) Immunohistochemical results were positive for CD99, EMA (focally) and a Ki67 value of $40 \%$, respectively. 